\title{
Nucleosome positioning on episomal human papillomavirus DNA in cultured cells
}

Isao Murakami

Keio University

Takashi Iwata ( $D$ isa00309@gmail.com )

Keio University

\section{Tohru Morisada}

Keio University

Kyoko Tanaka

Toho University Ohashi Medical Center

Daisuke Aoki

Keio University

\section{Research Article}

Keywords: Human papillomavirus, Nucleosome positioning, life cycle, long control region, E6

Posted Date: February 11th, 2021

DOl: https://doi.org/10.21203/rs.3.rs-153646/v1

License: (c) (1) This work is licensed under a Creative Commons Attribution 4.0 International License. Read Full License 


\section{Abstract}

Several human papillomaviruses (HPV) are associated with the development of cervical carcinoma. HPV DNA synthesis is increased during the differentiation of infected host keratinocytes as they migrate from the basal layer of the epithelium to the spinous layer, but the molecular mechanism is unclear. Nucleosome positioning affects various cellular processes such as DNA replication and repair by permitting the access of transcription factors to promoters to initiate transcription. In this study, nucleosome positioning on virus chromatin was investigated in normal immortalized keratinocytes (NIKS) stably transfected with HPV16 or HPV18 genomes to determine if there is an association with the viral life cycle. Micrococcal nuclease-treated DNA analyzed by Southern blotting using probes against HPV16 and HPV18 and quantified by nucleosome scanning analysis using real-time PCR revealed mononucleosomal-sized fragments of 140-200 base pairs that varied in their location within the viral genome according to whether the cells were undergoing proliferation or differentiation. Notably, changes in the regions around nucleotide 110 in proliferating and differentiating host cells were common to HPV16 and HPV18. Our findings suggest that change in nucleosome position on viral DNA during host cell differentiation is an important regulatory event in the viral life cycle.

\section{Introduction}

Papillomaviruses (PVs) are small double-stranded DNA viruses that infect stratified epithelia, causing benign or malignant hyperproliferative lesions with the completion of the human papillomavirus (HPV) life cycle, which is closely linked to the differentiation of keratinocytes. One of the most typical characteristics of PVs is their genotype-specific host restriction and the preference shown by different PV types for distinct anatomical sites ${ }^{1-3}$. To date, more than 200 HPV types have been sequenced and are further divided into low- and high-risk types based on the cancer risk associated with their infection ${ }^{4,5}$. Types $16,18,31,33,34,35,39,45,51,52,58$, and 70 are regarded as high-risk HPV types because they are found in cervical carcinomas. HPVs are thought to gain access to the basal cells of the epithelium through small traumas. The virus life cycle is linked to the differentiation of the infected cell as it migrates from the basal to the spinous layer of the epithelium. Both the replication of viral DNA and transcription from HPV promoters increase in response to differentiation of the host keratinocytes. However, the mechanism responsible for the increase in HPV DNA synthesis has not been clearly demonstrated.

The nucleosome is the basic unit of eukaryotic chromatin, consisting of a histone core around which DNA is wrapped. Each histone core is composed of two of each of the histone proteins $\mathrm{H} 2 \mathrm{~A}, \mathrm{H} 2 \mathrm{~B}, \mathrm{H} 3$, and $\mathrm{H} 4$. A stretch of approximately $147 \mathrm{bp}$ DNA wraps in 1.67 left-handed turns around the histone octamer to form a nucleosome. The combination of nucleosome positions and their chemical and compositional modifications is critical to genome regulation. The spatial accessibility of the nucleosome-protected sequence differs dramatically from that of the nucleosome-free sequence. Therefore, nucleosome positioning and distribution may affect DNA-related cellular processes such as DNA replication, repair, 
transcription, and recombination, and may be related to the regulation of gene expression and the local mutation rate ${ }^{6-8}$.

Transcription regulatory mechanisms have been found to play a role in the life cycle of viruses. This has been particularly well documented for the DNA genome of the retrovirus mouse mammary tumor virus (MMTV). The long terminal repeat (LTR) of MMTV is organized in the form of six nucleosomes. Mutual influences between one of these nucleosomes and certain transcription factors determine promoter access and transcriptional activation ${ }^{9,10}$. In the SV40, most of the regulatory region is normally free of nucleosomes ${ }^{11,12}$. Nucleosomes can be assembled in vitro that overlap with binding sites for the Sp1 transcription factor in the early promoter, with flanking nucleosomes possibly positioned as a result of neighboring effects ${ }^{13}$. Antagonistic interactions between nucleosomes and Sp1 may reduce the promoter access of the transcriptional apparatus ${ }^{14,15}$. Access of the replication machinery to the SV40 origin of replication is also blocked by nucleosomes, and this can be overcome by nucleosome remodeling in the presence of $T$ antigen ${ }^{16,17}$. In papillomaviruses, the chromatin organization has been established for bovine papillomavirus type 1 (BPV-1) in situ ${ }^{18,19}$, but whether nucleosomes persist on BPV-1 DNA packaged in viral particles remains unclear ${ }^{20}$. HPV genomes have been shown to be bound by nucleosomes in an ordered arrangement around the viral promoters ${ }^{21}$.

In this study, we used normal immortal keratinocyte (NIKS) cell lines containing HPV16 and HPV18 genomes as a cell model to compare the life cycle strategies of HPV. NIKS cells grown in a monolayer switch from a proliferative and undifferentiated proliferation mode at pre-confluence to a differentiated and post-mitotic proliferation mode at post-confluence. We report here the nucleosome positioning on HPV16 and HPV18 DNA during the maintenance and propagation of keratinocytes. These results provide insights into the control of the HPV life cycle from the long control region (LCR) to the E6 gene. This is the first study that has used normal keratinocytes harboring episomal HPV DNA to investigate nucleosome positioning.

\section{Results}

\section{Nucleosome organization of the HPV genome and host genome}

HPV16 or HPV18 genome chromatin was digested with 1/5 U to 5 U MNase. The DNA was electrophoretically separated on a $1.5 \%$ agarose gel and stained using SYBR Safe. The MNase-treated chromatin was examined by Southern blotting using probes for full-length HPV16 or HPV18 in transfected NIKS cells. Digestion of the HPV16 and HPV18 genomes using MNase resulted in a nonrandom cleavage pattern of approximately $200 \mathrm{bp}$ fragments. This finding suggested the presence of specifically localized or phased nucleosomes on HPV16 and HPV18 genome chromatin. Mononucleosome bands (approximately 200 bp) were most evident in the proliferating NIKS cells digested with $1 \mathrm{U}$ and $5 \mathrm{U}$ MNase and differentiating NIKS cells digested with $1 \mathrm{U}$ and $5 \mathrm{U}$ MNase. These bands were excised from the agarose gel and quantified using real-time PCR. 


\section{Nucleosome positioning on episomal HPV DNA in cultured cells}

We next performed a nucleosome-scanning experiment using real-time PCR and interpreted the results as follows. First, if a nucleosome was perfectly positioned, a primer pair located completely within this nucleosome would yield a maximal histone density that was defined as $100 \%$. However, if one primer was located within a nucleosome and the other primer was located outside the nucleosome, the apparent nucleosome density would be $0 \%$. Thus, a positioned nucleosomal array would generate peaks and valleys, with the valleys corresponding to linker regions between the nucleosomes. Second, a region occupied by randomly positioned nucleosomes would display a relatively constant level of nucleosome density that was less than the maximal level. Third, a region deficient in nucleosomes would display a low density over the entire region.

We used NIKS cells harboring HPV16 or HPV18 that were cultured under proliferating or differentiating conditions. In proliferating NIKS cells harboring HPV16, nt.11-240 and nt.311-570 of HPV16 DNA were relatively MNase-resistant (Figure 1A). In differentiating NIKS cells harboring HPV16, nt.11-150 and nt.191-330 of HPV16 DNA became more MNase-resistant, and nt.41-240 became less MNase-resistant. In proliferating NIKS cells harboring HPV18, nt.11-270 and nt.431-570 of HPV18 DNA were relatively MNase-resistant. In differentiating NIKS cells harboring HPV18, nt.41-300 of HPV18 DNA became less MNase-resistant (Figure 1B). Thus, the MNase-resistant regions of HPV DNA changed according to the proliferating or differentiating status of the host cell. Furthermore, a change in the MNase-resistant region around nt.110 between the proliferating and differentiating conditions of the host cell was common in HPV16 and HPV18 DNA.

\section{Discussion}

In this study, we analyzed nucleosome positioning on HPV DNA in NIKS cells harboring HPV16 or HPV18 cultured under proliferating or differentiating conditions. NIKS cells are spontaneously immortalized human keratinocyte cells arising from the BC-1-Ep strain of normal human neonatal foreskin keratinocytes ${ }^{24}$. NIKS cells, which are non-tumorigenic cells, maintain cell type-specific proliferation and differentiation characteristics in monolayer culture. We previously reported a cell model using NIKS cells harboring HPV11 and HPV16 to draw direct comparisons between the life cycle strategies of HPV that we used in the current paper ${ }^{25}$. In previous studies, we found that the pre-confluent state of NIKS cells exhibited the molecular properties of cell proliferation, while the post-confluent state had the molecular properties of cell differentiation. Therefore, NIKS cells represent an important new tool for the study of proliferation and differentiation in stratified squamous epithelia.

Standard MNase positioning can describe positioned nucleosomes, but it does not easily distinguish whether a given DNA region is occupied by no nucleosomes or randomly positioned nucleosomes. In addition, even when the positioned nucleosomes have been identified, it is difficult to specify the percentage of chromosomes that have a nucleosome at this position. Therefore, we modernized a nucleosome scanning method for positioning nucleosomes and identifying nucleosome density in a 
quantitative manner. Chromatin and purified genomic DNA were digested with MNase, and mononucleosomal-sized DNA fragments (140-200 bp) were isolated using gel electrophoresis. In the proliferating NIKS cells harboring HPV16, nt.11-240 and nt.311-570 of HPV16 DNA were relatively MNase-resistant. However, in the differentiating NIKS cells harboring HPV16, nt.11-150 and nt.191-330 of HPV16 DNA became more MNase-resistant, and nt.41-240 became less MNase-resistant. In proliferating NIKS cells harboring HPV18, nt.11-270 and nt.431-570 of HPV18 DNA were relatively MNase-resistant. In differentiating NIKS cells harboring HPV18, nt.41-300 of HPV18 DNA became less MNase-resistant. These results indicated that changes in the MNase-resistant regions around nt.110 between the proliferating and differentiating conditions of the host cell were common to HPV16 and HPV18. This change might represent a general modification that occurs among other HPV types and may serve as an important regulatory function during the viral life cycle that depends on the status of host cell differentiation. In differentiating cells, nt.41-240 of HPV16 and nt.41-300 of HPV18 DNA became less MNase-resistant, and this change may improve the accessibility of multiple proteins that interact directly with promoter DNA. In this study, we used episomal HPV16 and HPV18 ${ }^{25}$. This might be the reason that differences in MNase-resistant or less MNase-resistant areas were observed between HPV16 and HPV18. Most nucleosomes that exist in eukaryotic and viral chromatin are probably not specifically positioned ${ }^{26}$, and most often assume random positions by sliding freely along any particular DNA segment ${ }^{27,28}$. However, the sequences of particular nucleotide segments can affect physical properties such as their curvature, and these properties may determine the precise position of nucleosomes ${ }^{29,30}$. However, nucleosomes were found to occupy specific positions relative to regulatory DNA sequences in the majority of several carefully studied genes. For some of these genes, such as the LTR of MMTV $9,31,32$ and Xenopus 5S rRNA genes ${ }^{33}$, only one or a few nucleosomes are specifically positioned because of the underlying structural properties of the DNA, while neighboring nucleosomes may be arranged in a regular array relative to a specifically positioned nucleosome, thereby also binding a constant segment.

In conclusion, our results suggest that a shift in the position of the nucleosomes in the chromatin organization of HPV16 and HPV18 is an important regulatory event during the viral life cycle that depends on the status of host cell differentiation.

\section{Methods}

Cell culture

293T cells (ATCC) were maintained in Dulbecco's modified Eagle's medium (DMEM; Sigma, St. Louis, MO USA) supplemented with $10 \%$ fetal bovine serum (FBS; HyClone) and $1 \%$ penicillin and streptomycin. NIKS cells were maintained at subconfluence on mitomycin C-treated J2 3T3 feeder cells in F medium $\left(0.66 \mathrm{mM} \mathrm{Ca}^{2+}\right)$ composed of three parts $\mathrm{F}-12$ medium and one part DMEM supplemented with $5 \% \mathrm{FBS}$, insulin $(5 \mu \mathrm{g} / \mathrm{mL})$, cholera toxin $(8.4 \mathrm{ng} / \mathrm{mL})$, adenine $(24 \mu \mathrm{g} / \mathrm{mL})$, epidermal growth factor (10 $\mathrm{ng} / \mathrm{mL})$, and hydrocortisone $(0.4 \mu \mathrm{g} / \mathrm{mL})$, as previously described ${ }^{22}$. J2 3T3 feeder cells were removed from the culture dish by treatment with $0.02 \%$ EDTA. To remove the epithelial cells from the culture dish, 
the monolayer was rinsed with phosphate-buffered saline (PBS) followed by the addition of $0.1 \%$ trypsin/0.5 mM EDTA. The cells were then recovered from the suspension by multiple dilutions with $\mathrm{E}$ medium and cold PBS, followed by centrifugation.

\section{Generation of NIKS cell lines containing HPV genomes}

Plasmids containing HPV16 and HPV18 genomes were digested with BamH/or EcoR/to release the whole viral genome. The linearized HPV16 and HPV18 genomes were then re-circularized and purified as described previously ${ }^{23}$. Cells $\left(2 \times 10^{5}\right)$ were seeded in each well of a 6-well plate with incomplete F-media (no EGF). On the following day, the cells were transfected with $1600 \mathrm{ng}$ re-circularized HPV DNA and 400 ng pcDNA6 encoding a blasticidin resistance gene (Invitrogen) using FuGENE HD (Promega). The next day, the cells were seeded onto a $75 \mathrm{~cm}^{2}$ flask over blasticidin-resistant feeders with incomplete F-media. Cells were selected with $4 \mu \mathrm{g} / \mathrm{ml}$ blasticidin S with complete F-media (with $10 \mathrm{ng} / \mathrm{ml} \mathrm{EGF)} \mathrm{for} 4$ days and cultured for another $2-3$ days in the absence of blasticidin ${ }^{23}$.

The confluency of NIKS cells in culture strongly induces commitment to terminal differentiation 24,25 . Therefore, to induce differentiation, NIKS cells harboring HPV16 or HPV18 were cultured to post-confluent conditions and then collected for further analysis.

\section{Nuclei preparation}

For analysis of the chromatin organization of the HPV16 and HPV18 genomes, cultured NIKS cells harboring HPV16 and HPV18 were washed twice with ice-cold PBS and harvested with a rubber policeman. The pellet was centrifuged at $3000 \times \mathrm{g}$ for $5 \mathrm{~min}$ and washed with a buffer containing $10 \%$ sucrose, $60 \mathrm{mM} \mathrm{KCl}, 35 \mathrm{mM} \mathrm{HEPES}(\mathrm{pH} 7.4), 5 \mathrm{mM} \mathrm{KH}_{2} \mathrm{PO}_{4}, 5 \mathrm{mM} \mathrm{MgCl}_{2}$, and $0.5 \mathrm{mM} \mathrm{CaCl}_{2}$. The pellet was then suspended in a cold buffer containing $10 \%$ sucrose, $60 \mathrm{mM} \mathrm{KCl}, 35 \mathrm{mM}$ HEPES (pH 7.4), $5 \mathrm{mM}$ $\mathrm{KH}_{2} \mathrm{PO}_{4}, 5 \mathrm{mM} \mathrm{MgCl}_{2}, 3 \mathrm{mM} \mathrm{CaCl}_{2}$, and $15 \mathrm{mM} \mathrm{NaCl}$. After the addition of Nonidet P-40 (final concentration, $0.1 \%$ ), the samples were pipetted 10 times. To obtain genomic DNA, NIKS cells harboring HPV16 and HPV18 were washed twice with ice-cold PBS and harvested with a rubber policeman. The DNA was then purified using a QIAamp DNA Mini Kit (Qiagen, Hilden, Germany).

\section{Micrococcal nuclease (MNase) digestion of chromatin and purified DNA}

The DNA was digested with $1-5 \mathrm{U}$ MNase at $25^{\circ} \mathrm{C}$ for $5 \mathrm{~min}$. The reactions were stopped by the addition of $10 \mathrm{mM}$ EDTA, $1 \%$ SDS, and proteinase $\mathrm{K}(800 \mu \mathrm{g} / \mathrm{mL})$ at $37^{\circ} \mathrm{C}$ overnight. The DNA was then purified using phenol and phenol-chloroform extraction and ethanol precipitation.

\section{Southern blotting}

MNase-treated chromatin was examined using Southern blotting. The blot was probed with full-length HPV16 or HPV18 using a Phototope Kit (New England Biolabs, Ipswich, MA, USA). The DNA was loaded onto a $1.5 \%$ agarose gel in $0.5 \times \mathrm{TBE}$, and the gel was electrophoresed at $50 \mathrm{~V}$ for $3 \mathrm{~h}$. The 
electrophoretically separated DNA was transferred from the agarose gel to a nylon membrane (Hybond$\mathrm{N}+$; General Electric, $\mathrm{CT}$, USA) at room temperature overnight. Hybridization was conducted at $42^{\circ} \mathrm{C}$ overnight. The bands were detected by chemiluminescent detection and the Phototope-Star Detection Kit (New England Biolabs).

\section{Nucleosome scanning analysis}

MNase-treated chromatin and purified DNA samples were electrophoretically separated on a $1.5 \%$ agarose gel; mononucleosome-sized fragments were excised from the gel and purified. The resulting material was analyzed on the ViiA 7 Real-Time PCR System (Life Technologies) using Power SYBR Green/ROX master mix (Thermo Fisher Scientific) with 15 min denaturation at $95^{\circ} \mathrm{C}$, followed by 45 cycles of $95^{\circ} \mathrm{C}$ for $15 \mathrm{~s}$ and $60^{\circ} \mathrm{C}$ for $60 \mathrm{~s}$. The PCR amplicons were set every $30 \mathrm{bp}$ of the HPV genome, spanning the LCR and the E6 gene. Each amplicon was 140 bp long, including 30mer primers, and overlapped with its neighboring amplicons by 110 bp (Figure 2).

\section{Declarations}

\section{Additional information}

No potential conflict of interest relevant to this article was reported.

\section{Acknowledgments}

We thank Miyuki Saito and members of the Doorbar Lab for helpful advice. We also thank H. Nikki March, $\mathrm{PhD}$, and Gabrielle White Wolf, PhD, from Edanz Group (https://en-author-services.edanz.com/ac) for editing a draft of this manuscript.

Author contributions

Conceptualization: I.M., T.I.; data curation: I.M., T.I., T.M.; formal analysis: I.M.; methodology: I.M.; project administration: T.I., supervision: T.I., T.M., K.T., D.A.; writing-original draft: I.M.; writing-reviewing and edition: T.I., T.M., K.T., D.A. All authors discussed the results, commented on the manuscript, and gave final approval for publication.

\section{References}

1. Van Doorslaer K. Evolution of the papillomaviridae. Virology. 2013; 445(1-2):11-20.

2. Bravo IG, Felez-Sanchez M. Papillomaviruses: Viral evolution, cancer and evolutionary medicine. Evolution, medicine, and public health. 2015; 2015(1):32-51.

3. Chen Z, DeSalle R, Schiffman M, Herrero R, Wood CE, Ruiz JC, et al. Niche adaptation and viral transmission of human papillomaviruses from archaic hominins to modern humans. PLoS pathogens. 2018;14(11):e1007352. 
4. Doorbar J, Egawa N, Griffin H, Kranjec C, Murakami I. Human papillomavirus molecular biology and disease association. Reviews in medical virology. 2015; 25 Suppl 1:2-23.

5. Egawa N, Doorbar J. The low-risk papillomaviruses. Virus research. 2017; 231:119-27.

6. Cosma MP. Ordered recruitment: gene-specific mechanism of transcription activation. Mol Cell. 2002;10(2):227-36

7. Narlikar GJ, Fan HY, Kingston RE. Cooperation between complexes that regulate chromatin structure and transcription. Cell 2002;108(4):475-87.

8. Strahl BD, Allis CD. The language of covalent histone modifications. Nature 2000;6;403(6765):41-5.

9. Archer, T. K., M. G. Cordingley, R. G. Wolford, and G. L. Hager. 1991. Transcription factor access is mediated by accurately positioned nucleosomes on the mouse mammary tumor virus promoter. Mol. Cell. Biol. 11:688-698.

10. Truss, M., J. Bartsch, A. Schelbert, R. J. G. Hache, and M. Beato. 1995. Hormone induces binding of receptors and transcription factors to a rearranged nucleosome on the MMTV promoter in vivo. EMBO J. 14:1737-1751.

11. Batson, S. C., S. Rimsky, R. Sundseth, and U. Hansen. 1993. Association of nucleosome-free regions and basal transcription factors with in vivo-assembled chromatin templates active in vitro. Nucleic Acids Res. 21:3459-3468.

12. Kondoleon, S. K., N. A. Kurkinen, and L. M. Hallick. 1989. The SV40 nucleosome-free region is detected throughout the virus life cycle. Virology 173:129-135.

13. Jeong, S. W., and A. Stein. 1994. DNA sequence affects nucleosome ordering on replicating plasmids in transfected COS-1 cells and in vitro. J. Biol. Chem. 269:2197-2205.

14. Knezetic, J. A., and D. S. Luse. 1986. The presence of nucleosomes on a DNA template prevents initiation by RNA polymerase II in vitro. Cell 45:95-104.

15. Li, B., C. C. Adams, and J. L. Workman. 1994. Nucleosome binding by the constitutive transcription factor Sp1. J. Biol. Chem. 269:7756-7763.

16. Alexiadis, V., P. D. Varga-Weisz, E. Bonte, P. B. Becker, and C. Gruss. 1998. In vitro chromatin remodeling by chromatin accessibility complex (CHRAC) at the SV40 origin of DNA replication. EMBO J. 17:3428-3438.

17. Ramsperger, U., and H. Stahl. 1995. Unwinding of chromatin by the SV40 large T antigen DNA helicase. EMBO J. 14:3215-3225.

18. Rosl, F., W. Waldeck, H. Zentgraf, and G. Sauer. 1986. Properties of intracellular bovine papillomavirus chromatin. J. Virol. 58:500-507.

19. Waldeck, W., F. Ro"sl, and H. Zentgraf. 1984. Origin of replication in episomal bovine papilloma virus type 1 DNA isolated from transformed cells. EMBO J. 3:2173-2178.

20. Larsen, P. M., L. Storgaard, and S. J. Fey. 1987. Proteins present in bovine papillomavirus particles. J. Virol. 61:3596-3601. 
21. del Mar Pena LM, Laimins LA. Differentiation-dependent chromatin rearrangement coincides with activation of human papillomavirus type 31 late gene expression. J Virol 2001;75(20):10005-13.

22. Flores ER, Allen-Hoffmann BL, Lee D, Sattler CA, Lambert PF. Establishment of the human papillomavirus type 16 (HPV-16) life cycle in an immortalized human foreskin keratinocyte cell line. Virology.1999;262(2):344-54.

23. Egawa N, Wang Q, Griffin HM, Murakami I, Jackson D, Mahmood R, et al. HPV16 and 18 genome amplification show different E4-dependence, with 16E4 enhancing E1 nuclear accumulation and replicative efficiency via its cell cycle arrest and kinase activation functions. PLoS pathogens. 2017;13(3):e1006282.

24. Poumay $Y$ and Pittelkow M. Cell Density and Culture Factors Regulate Keratinocyte Commitment to Differentiation and Expression of Suprabasal KI/KIO Keratins. J Invest Dermatol. 1995;104:271-276.

25. Murakami I, Egawa N, Griffin H, Yin W, Kranjec C, Nakahara T, Kiyono T, Doorbar J. Roles for E1independent replication and E6-mediated p53 degradation during low-risk and high-risk human papillomavirus genome maintenance. PLoS pthogens. 2019;15(5):e1007755.

26. Simpson, R. T. 1990. Nucleosome positioning can affect the function of a cis-acting DNA element in vivo. Nature 343:387-389.

27. Meersseman, G., S. Pennings, and E. M. Bradbury. 1992. Mobile nucleosomes, a general behavior. EMBO J. 11:2951-2959.

28. Pazin, M. J., P. Bhargava, E. P. Geiduschek, and J. T. Kadonaga. 1997. Nucleosome mobility and the maintenance of nucleosome positioning. Science 276:809-812.

29. FitzGerald, P. C., and R. T. Simpson. 1985. Effects of sequence alterations in a DNA segment containing the 5S RNA gene from Lythechinus variegatus on positioning of a nucleosome core particle in vitro. J. Biol. Chem. 260:15318-15324.

30. Hayes, J. J., T. D. Tullius, and A. P. Wolffe. 1990. The structure of DNA in a nucleosome. Proc. Natl. Acad. Sci. USA 87:7405-7409.

31. Chavez, S., and M. Beato. 1997. Nucleosome-mediated synergism between transcription factors on the mouse mammary tumor virus promoter. Proc. Natl. Acad. Sci. USA 94:2885-2890.

32. Richard-Foy, H., and G. L. Hager. 1987. Sequence-specific positioning of nucleosomes over the steroid-inducible MMTV promoter. EMBO J. 6:2321-2328.

33. Sera, T., and A. P. Wolffe. 1998. Role of histone $\mathrm{H} 1$ as an architectural determinant of chromatin structure and as a specific repressor of transcription on Xenopus 5S rRNA genes. Mol. Cell. Biol. 18:3668-3680.

\section{Figures}


Fig 1

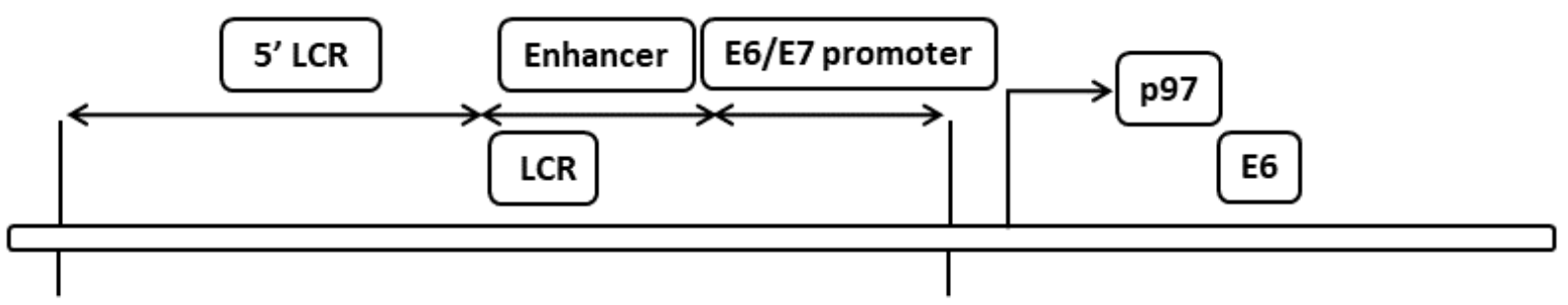

HPV 167157

HPV 187137

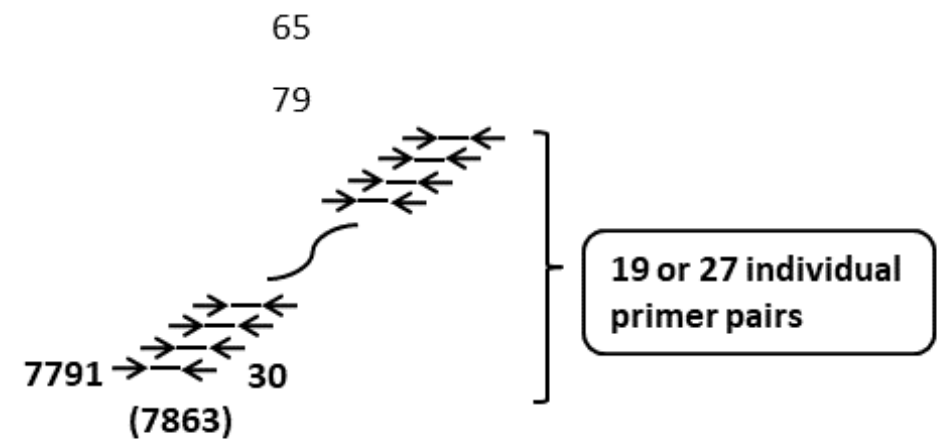

\section{Figure 1}

Nucleosome positioning on episomal HPV16/18 DNA in cultured cells. Chromatin and purified genomic DNA were digested with MNase, and mononucleosomal-sized DNA fragments (140-200 bp) were isolated using gel electrophoresis. The mononucleosomal-sized DNA fragments were quantified by realtime PCR. In proliferating NIKS cells harboring HPV16, nt.11-240 and nt.311-570 of HPV16 DNA were relatively MNase-resistant. In differentiating NIKS cells harboring HPV16, nt.11-150 and nt.191-330 of HPV16 DNA became more MNase-resistant and nt.41-240 became less MNase-resistant (A). In proliferating NIKS cells harboring HPV18, nt.11-270 and nt.431-570 of HPV18 DNA were relatively MNase-resistant. In differentiating NIKS cells harboring HPV18, nt.41-300 of HPV18 DNA became less MNase-resistant (B). 
Fig 2

A SYBR Safe

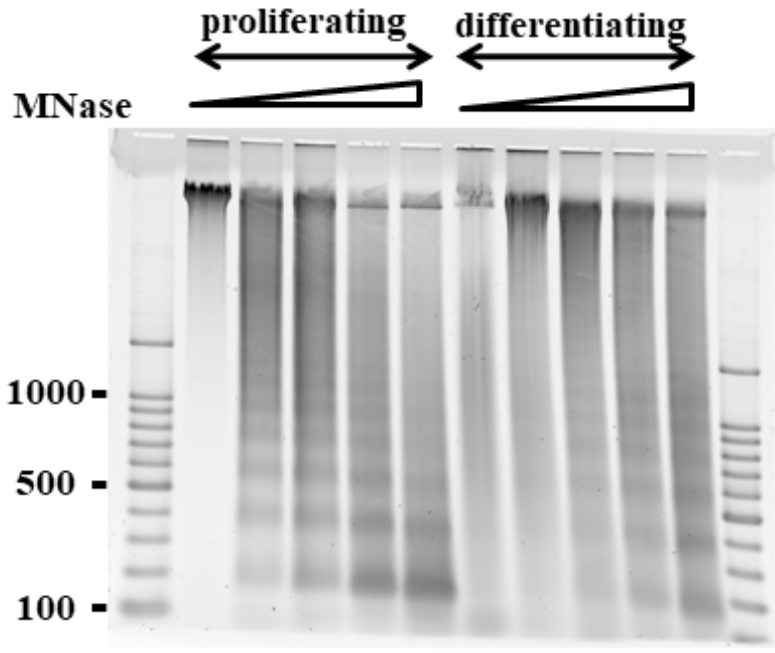

\section{B Southern blotting}

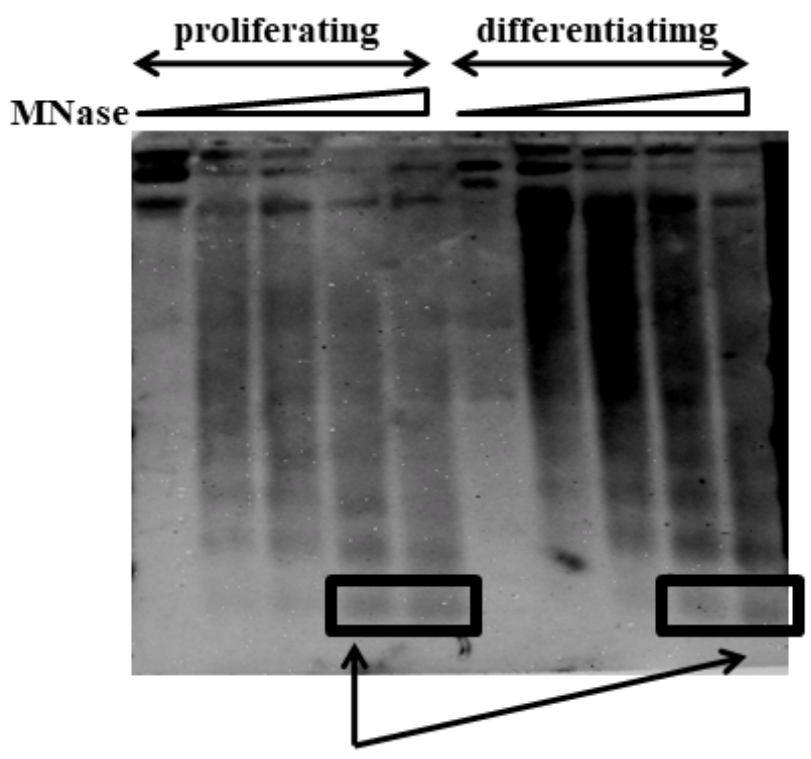

excision of the DNA band from a agarose gel

and quantification of by real-time PCR

\section{Figure 2}

Structure of HPV16/18 the long control region (LCR) and in E6. PCR amplicons were set every $30 \mathrm{bp}$ of the HPV genome spanning the LCR and E6 gene. Each amplicon was $140 \mathrm{bp}$ long including 30mer primers, and the neighboring amplicons overlapped by $110 \mathrm{bp}$. 
Fig 3

A

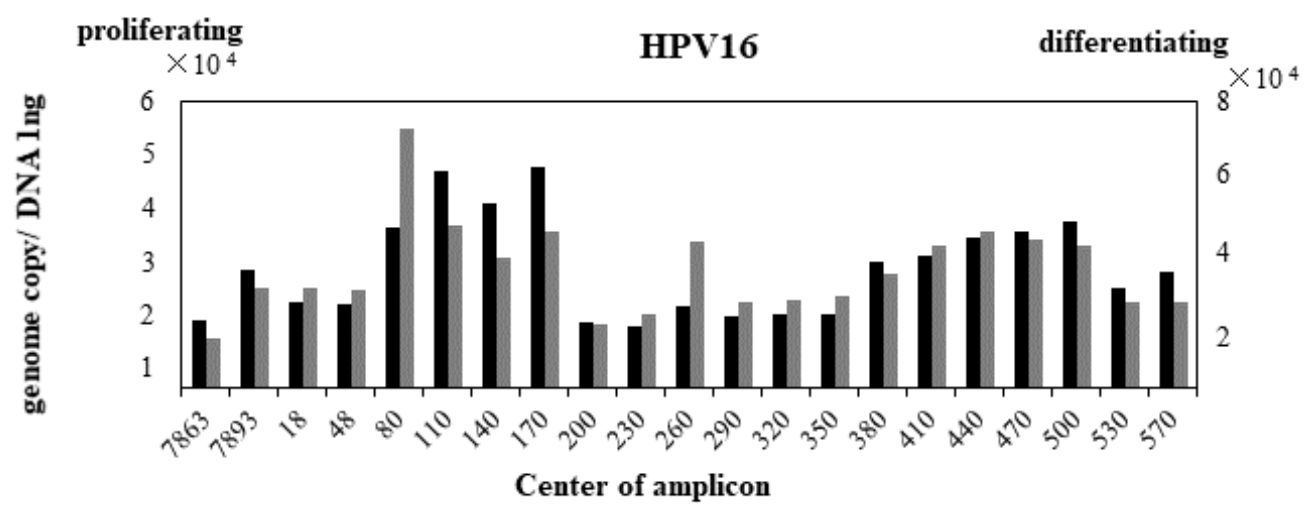

proliferating

differentiating

B

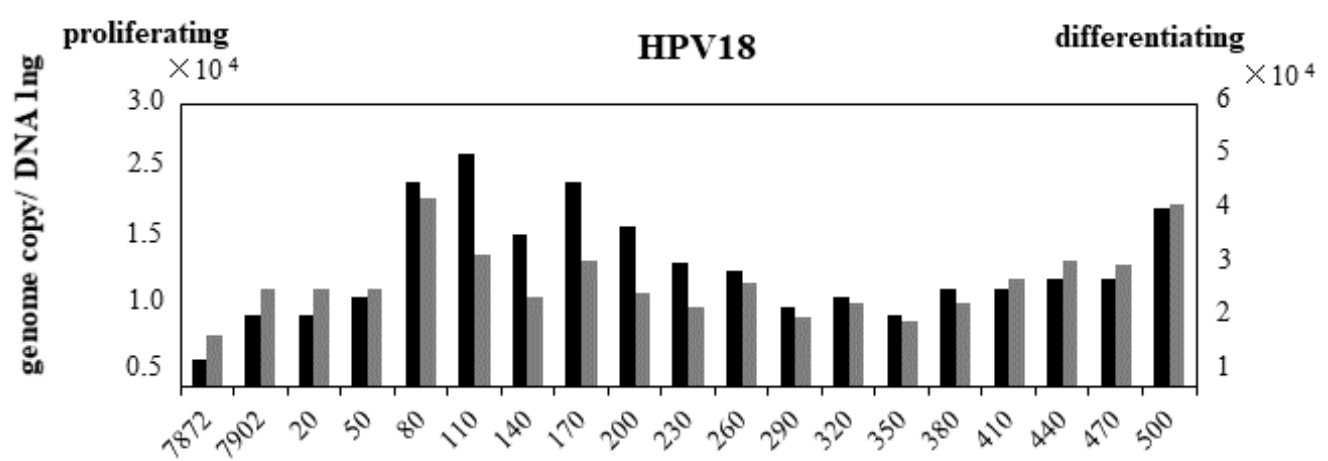

Center of amplicon

proliferating

differentiating

Figure 3

Nucleosome positioning on episomal HPV16/18 DNA in cultured cells. Chromatin and purified genomic DNA were digested with MNase, and mononucleosomal-sized DNA fragments (140 -200 bp) were isolated using gel electrophoresis. And then the mononucleosomal-sized DNA fragment was quantified by real-time PCR. In proliferating NIKS cells harboring HPV16, nt.11 $\neg-240$ and nt.311 -570 of HPV16 DNA were relatively MNase-resistant. In differentiating NIKS cells harboring HPV16, nt.11 $\neg-150$ and nt.191 $\neg-330$ of HPV16 DNA became more MNase-resistant, and nt.41 -240 became less MNaseresistant (A). In proliferating NIKS cells harboring HPV18, nt.11 $\neg-270$ and nt.431 -570 of HPV18 DNA were relatively MNase-resistant. In differentiating NIKS cells harboring HPV18, nt.41 -300 of HPV18 DNA became less MNase-resistant (B). 